\title{
(1) Coplo Chemistry \\ QSAR STUDIES OF SULPHONAMIDES AS CARBONIC ANHYDRASE II INHIBITORS USING TOPOLOGICAL INDICES
}

\author{
Prabhanand Patel Department Of Chemistry, A.P.S. university, Rewa-486003, India \\ Shailja Sachan* \\ Department Of Chemistry, Govt. M.S.G. college, Rewa-486001, India \\ *Corresponding Author
}

\section{Bhupendra Kumar Verma}

Balendra Singh

Pratiksha Patel

Santosh Tiwari
Department Of Chemistry, A.P.S. university, Rewa-486003, India

Department Of Chemistry, A.P.S. university, Rewa-486003, India

Department Of Chemistry, A.P.S. university, Rewa-486003, India

Department Of Chemistry, A.P.S. university, Rewa-486003, India

ABSTRACT In this paper topological indices have been used in modeling log KI activity of 47 organic compounds acts as carbonic anhydrase II inhibitors. The 7-parametric model gave excellent results which contains mixed parameters. The results are discussed using different statistical parameters.

\section{KEYWORDS : Qsar Modeling, Log Ki, Topological Indices}

\section{INTRODUCTION}

Sulphonamide inhibitors of Carbonic anhydrase are extensively used in clinical medicine and as diagnostic species, their main applications being in the treatment of glaucoma, macular edema, epilepsy, bronchitis, pneumonia and other neurological disorders. Carbonic anhydrase inhibition of sulfanilamide discovered by Mann and Keilin has led to important drugs such as the sulfamides with CA inhibitory properties. Several such drugs are presently available, such as the recently introduced topical sulfonamides dorzolamide and brinzolamide, in addition to the classical, systemically acting inhibitors acetazolamide, methazolamide, ethoxzolamide, and dichlorophenamide which have been adopted clinically for more than 45 years $^{1-3}$.

Sulfonamide CAIs derivatives of simple aromatic or heterocyclic aromatic sulfonamides have already shown with excellent CA inhibitory properties against many CA isozymes isolated so far in diverse organisms. Many drug derivatives of heterocyclic and aromatic classes of sulfonamides have been modelled, synthesized and investigated for their biological activity ${ }^{4}$.

The aromatic/heterocyclic sulfonamides act as carbonic anhydrase inhibitors and other types of derivatives show diuretic activity, hypoglycaemic activity, anticancer properties or may act as inhibitors of the aspartic HIV protease being used for the treatment of AIDS and HIV infection among other During the last few years, Supuran and his co-workers has deeply studied different aromatic sulfonamides as most effective carbonic anhydrase II inhibitors ${ }^{5}$. Since weaker CAIs as compared to heterocyclicones many aromatic substituted sulfonamides were used to be strong inhibitors with low KI values within the nanomolar range. Special attention was paid to aromatic sulphonamides substituted at the para position as they exhibit higher affinity with the zinc enzyme compared to an orthosubstituted aromatic sulfonamide. This may be due to the steric impairment of the ortho -substituent for the binding of such compounds to the $\mathrm{Zn}$ (II) ion within the enzyme active site.

Due to the biological importance of sulfonamides as potent CAIs, quantitative structure-activity/property relationship (QSAR/QSPR) models have been proposed for the modelling and prediction of CA inhibitory activity of different aromatic and heterocyclic aromatic sulfonamides using different molecular, topological descriptors. QSARs are mathematical relationships between a set of descriptors and the biological activity of the system being studied. QSAR models can be used as a useful tool in drug design, as they have the potential to shorting out the time and effort required to develop new compounds by reducing expensive and time-consuming trial-and-error experiments ${ }^{6-8}$. Our objective is to model a fast and reliable method to predict the CA inhibition activity of sulfonamides. The sulfonamides used in associate hydrazine moieties, ureas, sulfureas or simple aliphatic derivatives. The proposed model can be used as a first step for the formulation of an optimization problem from which the best parasubstituent will be derived.

For this QSAR analysis we have selected a list of 47 para-substituted sulfonamides from the work of Melagraki and co-workers ${ }^{6}$. The aromatic sulfonamides are presented in Scheme 1. The inhibition data are expressed in erms of nanomolar affinity $(\mathrm{KI})$ for the investigated isozyme. First, the chemical structures were designed using Chemsketch19 and were saved as .mol files. We have used Dragon software to calculate the topological indices data of 11 descriptors shown in Table 1, which are related to the arrangements of atoms in compounds as no. \& types, of atoms, branching and no of multiple bonds etc ${ }^{9-14}$. Topological indices are a common useful tool for QSAR/QSPR taking into account their simplicity and rapidity of computation. This is particularly valuable now as one can analyze structures used for QSAR studies prior to any high throughput synthesis and testing.

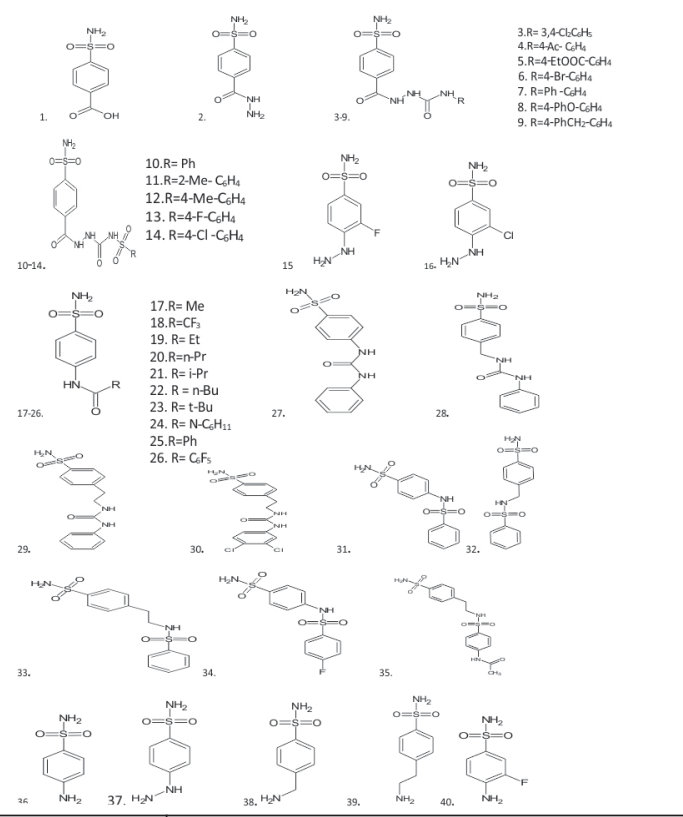

\begin{tabular}{c|c}
\hline Submitted : $22^{\text {nd }}$ August, 2019 & Accepted : 11 \\
\hline
\end{tabular}




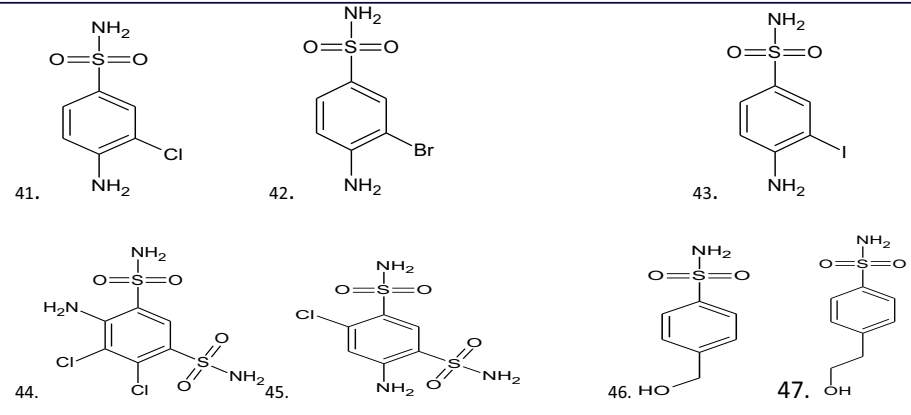

Scheme No.1: Structure of Sulphonamide Derivatives

Table 1 : Calculated Descriptors and Carbonic Anhydrase II Inhibitors $\left(\log K_{\mathrm{i}}\right)$

\begin{tabular}{|c|c|c|c|c|c|c|c|c|c|c|c|c|}
\hline Comp. No. & $\log \mathrm{KI}$ & FW & $\mathrm{Xt}$ & $\mathrm{X} 3$ & $\mathrm{X} 0 \mathrm{v}$ & XMOD & ZM1Mad & ZM1Per & ZM2Mad & Pol & piPC03 & piPC05 \\
\hline 1 & 2.4116 & 201.19 & 0.366 & 3.939 & 6.805 & 46.806 & 106.969 & 445.73 & 118.265 & 17 & 3.932 & 4.673 \\
\hline 2 & 2.0934 & 215.22 & 0.35 & 4.445 & 7.291 & 50.368 & 110.837 & 468.72 & 124.046 & 19 & 3.989 & 4.71 \\
\hline 3 & 1.1139 & 403.24 & 0.25 & 8.359 & 13.914 & 88.998 & 205.67 & 846.47 & 222.378 & 36 & 4.564 & 5.361 \\
\hline 4 & 1.1761 & 394.4 & 0.24 & 8.423 & 13.463 & 89.94 & 189.163 & 866.57 & 206.799 & 37 & 4.477 & 5.247 \\
\hline 5 & 0.9542 & 408.4 & 0.235 & 9.051 & 13.963 & 93.168 & 193.163 & 889.61 & 212.464 & 39 & 4.511 & 5.257 \\
\hline 6 & 0.8633 & 413.24 & 0.253 & 7.787 & 13.611 & 88.554 & 234.502 & 784.5 & 215.412 & 33 & 4.5 & 5.313 \\
\hline 7 & 1.0414 & 412.46 & 0.223 & 9.86 & 14.647 & 95.618 & 196.631 & 868.77 & 221.148 & 42 & 4.71 & 5.553 \\
\hline 8 & 1.2553 & 428.46 & 0.219 & 10.01 & 15.055 & 99.333 & 204.838 & 919.25 & 228.135 & 42 & 4.7 & 5.513 \\
\hline 9 & 1.1761 & 426.48 & 0.219 & 10.01 & 15.147 & 98.517 & 200.631 & 891.81 & 224.148 & 42 & 4.7 & 5.513 \\
\hline 10 & 1.8261 & 336.36 & 0.257 & 7.377 & 11.647 & 77.82 & 162.631 & 722.53 & 180.148 & 31 & 4.369 & 5.112 \\
\hline \begin{tabular}{|l|}
11 \\
\end{tabular} & 1.7324 & 412.4 & 0.243 & 8.906 & 14.188 & 96.872 & 227.486 & 902.05 & 261.715 & 41 & 4.682 & 5.416 \\
\hline 12 & 0.9912 & 412.4 & 0.243 & 8.912 & 14.188 & 96.77 & 227.486 & 902.05 & 259.045 & 40 & 4.673 & 5.438 \\
\hline 13 & 0.9777 & 416.4 & 0.243 & 8.912 & 14.066 & 97.637 & 231.316 & 940.66 & 261.955 & 40 & 4.673 & 5.438 \\
\hline 14 & 0.959 & 432.8 & 0.243 & 8.912 & 14.822 & 99.946 & 243.005 & 940.66 & 268.804 & 40 & 4.673 & 5.438 \\
\hline 15 & 1.7076 & 205.2 & 0.366 & 4.096 & 6.761 & 47.657 & 108.453 & 460.53 & 121.752 & 18 & 3.932 & 4.727 \\
\hline 16 & 1.8808 & 221.66 & 0.366 & 4.096 & 7.517 & 49.966 & 120.142 & 460.53 & 130.199 & 18 & 3.932 & 4.727 \\
\hline 17 & 2.3909 & 268.21 & 0.324 & 5.237 & 8.478 & 59.054 & 145.826 & 644.88 & 154.781 & 23 & 4.043 & 4.754 \\
\hline 18 & 2.1239 & 268.21 & 0.324 & 5.237 & 8.478 & 59.054 & 145.826 & 644.88 & 154.781 & 23 & 4.043 & 4.754 \\
\hline 19 & 2.3655 & 228.26 & 0.336 & 4.504 & 7.844 & 52.764 & 114.809 & 480.75 & 126.436 & 19 & 3.932 & 4.7 \\
\hline 20 & 2.356 & 226.25 & 0.317 & 5.1 & 7.844 & 52.971 & 122.809 & 488.43 & 139.603 & 22 & 4.111 & 5.075 \\
\hline 21 & 2.4116 & 242.29 & 0.329 & 4.915 & 8.344 & 55 & 120.809 & 504.11 & 133.935 & 21 & 3.989 & 4.727 \\
\hline 22 & 2.3304 & 256.32 & 0.313 & 4.858 & 8.844 & 58.764 & 122.809 & 526.83 & 134.436 & 21 & 3.97 & 4.736 \\
\hline 23 & 2.3617 & 256.3 & 0.324 & 5.237 & 8.844 & 56.804 & 128.809 & 527.79 & 143.434 & 23 & 4.043 & 4.754 \\
\hline 24 & 1.7993 & 270.3 & 0.303 & 5.108 & 9.344 & 61.764 & 126.809 & 549.87 & 138.436 & 22 & 3.989 & 4.745 \\
\hline 25 & 1.5682 & 276.29 & 0.28 & 6.231 & 9.844 & 64.971 & 138.809 & 580.59 & 155.935 & 26 & 4.369 & 5.204 \\
\hline 26 & 1.2304 & 366.2 & 0.26 & 8.965 & 11.734 & 81.723 & 187.963 & 890.44 & 218.66 & 42 & 4.73 & 5.648 \\
\hline 27 & 2.3802 & 291.32 & 0.273 & 6.268 & 10.291 & 68.278 & 145.029 & 616.39 & 160.268 & 26 & 4.331 & 5.165 \\
\hline 28 & 2.0212 & 305.35 & 0.266 & 6.6 & 10.791 & 71.324 & 148.696 & 639.35 & 164.101 & 27 & 4.344 & 5.159 \\
\hline 29 & 1.8751 & 319.37 & 0.26 & 6.841 & 11.291 & 74.324 & 152.696 & 662.39 & 167.935 & 28 & 4.357 & 5.142 \\
\hline 30 & 1.1139 & 388.26 & 0.253 & 7.823 & 13.559 & 85.502 & 195.735 & 786.33 & 210.164 & 33 & 4.466 & 5.273 \\
\hline 31 & 1.6902 & 312.36 & 0.277 & 6.597 & 10.977 & 75.517 & 185.449 & 666.59 & 213.005 & 29 & 4.466 & 5.308 \\
\hline 32 & 1.6021 & 326.39 & 0.27 & 6.979 & 11.477 & 78.563 & 189.116 & 689.55 & 215.169 & 30 & 4.477 & 5.273 \\
\hline 33 & 1.4472 & 340.41 & 0.264 & 7.215 & 11.977 & 81.563 & 193.116 & 712.59 & 219.002 & 31 & 4.489 & 5.236 \\
\hline 34 & 0.9542 & 330.35 & 0.273 & 7.007 & 11.355 & 78.746 & 195.28 & 728.56 & 222.916 & 31 & 4.511 & 5.375 \\
\hline 35 & 1.8751 & 397.46 & 0.246 & 8.262 & 13.832 & 93.275 & 217.384 & 841.85 & 243.996 & 37 & 4.595 & 5.394 \\
\hline 36 & 2.4771 & 172.2 & 0.397 & 3.241 & 5.936 & 40.476 & 93.568 & 362.66 & 103.776 & 13 & 3.738 & 4.489 \\
\hline 37 & 2.5051 & 187.21 & 0.377 & 3.649 & 6.383 & 44.327 & 98.622 & 398.56 & 109.997 & 15 & 3.807 & 4.554 \\
\hline 38 & 2.2304 & 186.23 & 0.377 & 3.649 & 6.436 & 43.769 & 97.235 & 385.62 & 108.609 & 15 & 3.807 & 4.554 \\
\hline 39 & 2.2041 & 200.25 & 0.359 & 3.768 & 6.936 & 46.769 & 101.235 & 408.66 & 112.443 & 16 & 3.829 & 4.595 \\
\hline 40 & 1.7782 & 190.19 & 0.385 & 3.823 & 6.314 & 43.806 & 103.399 & 424.63 & 115.531 & 16 & 3.871 & 4.673 \\
\hline 41 & 2.0414 & 206.64 & 0.385 & 3.823 & 7.07 & 46.116 & 115.087 & 424.63 & 123.978 & 16 & 3.871 & 4.673 \\
\hline 42 & 1.6021 & 251.1 & 0.385 & 3.823 & 7.9 & 51.312 & 165.439 & 424.63 & 146.8 & 16 & 3.871 & 4.673 \\
\hline 43 & 1.8451 & 298.1 & 0.385 & 3.823 & 8.471 & 56.508 & 248.477 & 424.63 & 170.933 & 16 & 3.871 & 4.673 \\
\hline 44 & 1.4472 & 320.17 & 0.334 & 5.661 & 10.692 & 71.782 & \begin{tabular}{|l|l|}
199.147 \\
\end{tabular} & 676.81 & 232.389 & 29 & 4.317 & 5.242 \\
\hline 45 & 1.8751 & 305.15 & 0.342 & 4.85 & 10.245 & 68.928 & 192.121 & 642.6 & 217.167 & 25 & 4.22 & 5.081 \\
\hline 46 & 2.0969 & 187.21 & 0.377 & 3.649 & 6.397 & 44.123 & 97.98 & 398.41 & 109.272 & 15 & 3.807 & 4.554 \\
\hline 47 & 2.0414 & 201.24 & 0.359 & 3.768 & 6.897 & 47.123 & 101.98 & 421.45 & 112.94 & 16 & 3.829 & 4.595 \\
\hline
\end{tabular}

The descriptors have been used to compute, for highest correlation coefficient value it is necessary to decide which ones will be used. Among the 11 indices the selection of the best combinations was made with the use of an Elimination Selection Stepwise Regression (ESSWR) algorithm that was developed inhouse. The aim of variable subset selection is to reach optimal model complexity in predicting a response variable by a reduced set of descriptors that are not highly intercorrelated having multicollinearity. The descriptors that after shorting out giving the attachment, position, multiplicity of attaching groups and mean information content based on the vertex degree equality and the edge equality both, taken for the correlation analysis to model the potent sulfonamide drug. The DRAGON software for testing of best product has been used for the collection of the data of compounds study for the nature of attaching groups. This software offers several hundreds of descriptors from different perspectives relating to empirical, constitutional and topological indices characteristic to the molecules under multi-descriptor class environment ${ }^{15-16}$. We have been drawn the structure of the studied 
Volume-9 | Issue-12 | December - 2019 | PRINT ISSN No. 2249 - 555X | DOI : 10.36106/ijar

compounds in $\mathrm{ACD} / \mathrm{Chemsketch}$ using the standard procedure. The development in QSAR studies by using NCSS software.

CP-MLR is a 'filter' based variable selection procedure for model

\begin{tabular}{|c|c|c|c|c|c|c|c|c|}
\hline \multicolumn{9}{|c|}{ Table 2: Results of Regression Analysis } \\
\hline S. No. & Parameters Used & $\operatorname{Ai}(1 \ldots 3)$ & Intercept & $\mathrm{SE}$ & R2 & AR2 & $\mathrm{R} 2 \mathrm{CV}$ & F-Ratio \\
\hline 1 & FW & -0.005 & 3.2386 & 0.2985 & 0.6651 & 0.6577 & 0.637 & 89.385 \\
\hline \multirow[t]{2}{*}{2} & FW, & -0.007 & & & & & & \\
\hline & $\mathrm{XT}$ & -3.3756 & 4.8853 & 0.2915 & 0.6879 & 0.6737 & 0.6422 & 48.49 \\
\hline \multirow[t]{3}{*}{3} & FW & -0.0087 & & & & & & \\
\hline & $\mathrm{Xt}$ & -4.5557 & & & & & & \\
\hline & Zmimad & 0.0019 & 5.4483 & 0.2937 & 0.6903 & 0.6687 & 0.621 & 31.95 \\
\hline \multirow[t]{4}{*}{4} & FW & -0.0094 & & & & & & \\
\hline & $\mathrm{Xt}$ & -8.6849 & & & & & & \\
\hline & ZM1Mad & 0.0045 & & & & & & \\
\hline & piPC05 & -0.8474 & 10.7398 & 0.2788 & 0.7274 & 0.7014 & 0.6631 & 28.018 \\
\hline \multirow[t]{5}{*}{5} & FW & -0.0139 & & & & & & \\
\hline & $\mathrm{Xt}$ & -9.6682 & & & & & & \\
\hline & ZM1Mad & 0.0078 & & & & & & \\
\hline & piPC05 & -1.328 & & & & & & \\
\hline & Pol & 0.0372 & 13.2771 & 0.2769 & 0.7375 & 0.7055 & 0.6577 & 23.041 \\
\hline \multirow[t]{6}{*}{6} & FW & -0.0115 & & & & & & \\
\hline & $\mathrm{Xt}$ & -11.2816 & & & & & & \\
\hline & ZM1Mad & 0.0057 & & & & & & \\
\hline & piPC05 & -1.2988 & & & & & & \\
\hline & Pol & 0.074 & & & & & & \\
\hline & X3 & -0.2653 & 13.8923 & 0.2746 & 0.7482 & 0.7104 & 0.6354 & 19.805 \\
\hline \multicolumn{9}{|c|}{ After deleting Com. No.24 and 27} \\
\hline \multirow[t]{6}{*}{7} & FW & -0.011 & & & & & & \\
\hline & $\mathrm{Xt}$ & -11.6539 & & & & & & \\
\hline & Zmimad & 0.0055 & & & & & & \\
\hline & piPC05 & -1.6171 & & & & & & \\
\hline & Pol & 0.0966 & & & & & & \\
\hline & $\mathrm{X} 3$ & -0.3461 & 15.3604 & 0.2571 & 0.7828 & 0.7485 & 0.6775 & 22.82 \\
\hline
\end{tabular}

Model No.1

$\log K_{\mathrm{I}}=3.2386( \pm 0.1634)-0.0049( \pm 0.0005) * \mathrm{FW}$

$$
\mathrm{n}=47 \quad \mathrm{r}^{2}=0.8155 \quad \mathrm{Se}=0.2985 \quad \text { F-Ratio }=89.39
$$

Here and thereafter, $\mathrm{n}=$ number of data point, $\mathrm{R}^{2}=$ regression coefficient, $\mathrm{Se}=$ Standard Error of estimation, $\mathrm{F}=$ Fischer statistics. As the coefficient of FW in Model No.1 is negative, the CA inhibition increases with a decrease in the value of FW. The value of correlation coefficient $r=0.9030$, which means that it can predict $81.55 \%$ variance ofCA inhibition.

\section{Model No.2}

$\log K_{\mathrm{I}}=4.8853( \pm 0.9331)-0.0070( \pm 0.0012) * \mathrm{FW}-$

$3.3756( \pm 1.8847) * \mathrm{Xt}$

$\mathrm{n}=47 \quad \mathrm{r}=0.8293 \quad \mathrm{Se}=0.2951 \quad \mathrm{~F}-$ Ratio $=48.49$

Topological indices are numerical quantifier of molecular topology and are sensitive to bonding pattern, symmetry, content of heteroatom as well as degree of complexity of atomic neighborhoods. Since structure of a compound depends derived from information based upon connectivity can reveal the role of structural or sub-structural information of a molecules in estimating biological activity. Further we have obtained various multiple regression model by increasing no. of descriptors in which model no. 7 is significant.

\section{Model No.7}

$\log K_{I}=15.3604( \pm 3.1193)-0.011( \pm 0.0047) * F W-11.6539$ $( \pm 3.3579) * \mathrm{Xt}$

$+0.0055( \pm 0.0042) * \mathrm{ZM} 1 \mathrm{Mad}-1.6171( \pm 0.4967) * \mathrm{piPC} 05$

$+0.0966( \pm 0.0390) *$ Pol - $0.3461( \pm 0.1999) * \mathrm{X} 3$

$\mathrm{n}=45 \quad \mathrm{r}^{2}=0.8847 \quad \mathrm{Se}=0.2571 \quad \mathrm{~F}-$ Ratio $=22.82$

In this study we have calculate correlation by cross validation method of 7 models, out of which model no 7 (hexa parametric) with correlation coefficient $r=0.9058$, and low press error leads to the development of statistically significant QSAR model, which allows understanding of the molecular properties/features that play an important role in governing the variation in the activities. In addition, this QSAR study allowed investigating influence of very simple and easy to compute descriptors in determine biological activities which could shed light on the key factors that may aid in design of novel potent molecules..

The randomization test suggests that the developed model have a probability of less than $1 \%$ that the model is generated by chance. The plot of observed vs predicted activity is shown in Fig. From the plot it can be sheen that MLR model is able to predict the activity of training set quit well (all Points are close to regression line) as well as external.

\section{CONCLUSION}

In view of result and discussions, we conclude that topological descriptor FW,Xt, ZM1Mad, piPC05, Pol and X3 can be successfully used for the QSAR study of sulphonamides as carbonic anhydrase II inhibitors using topological indices as potent antimicrobial drugs. These results will help medical as well as agriculture scientists in the design and prediction of new sulfonamide drugs exhibiting better activities than these reported in this result.

Table 3: Predicted $\log K_{\mathrm{i}}$ and Observed $\log \mathrm{K}_{\mathrm{i}}$ with Residual

\begin{tabular}{|l|l|l|l|}
\hline Comp. No. & Observed $\log \mathbf{K}_{\mathbf{I}}$ & Predicted $\log \mathbf{K}_{\mathbf{I}}$ & Residual \\
\hline 1 & 2.41 & 2.20 & 0.20 \\
\hline 2 & 2.09 & 2.21 & -0.12 \\
\hline 3 & 1.11 & 1.08 & 0.02 \\
\hline 4 & 1.17 & 1.46 & -0.28 \\
\hline 5 & 0.95 & 1.35 & -0.39 \\
\hline 6 & 0.86 & 1.08 & -0.22 \\
\hline 7 & 1.04 & 0.99 & 0.04 \\
\hline 8 & 1.25 & 0.92 & 0.32 \\
\hline 9 & 1.17 & 0.92 & 0.25 \\
\hline 10 & 1.82 & 1.75 & 0.06 \\
\hline 11 & 1.73 & 1.39 & 0.34 \\
\hline 12 & 0.99 & 1.25 & -0.26 \\
\hline 13 & 0.97 & 1.23 & -0.25 \\
\hline 14 & 0.95 & 1.11 & -0.15 \\
\hline 15 & 1.70 & 2.12 & -0.41 \\
\hline 16 & 1.88 & 2.00 & -0.12 \\
\hline
\end{tabular}




\begin{tabular}{|c|c|c|c|}
\hline 17 & 2.39 & 2.17 & 0.21 \\
\hline 18 & 2.12 & 2.17 & -0.05 \\
\hline 19 & 2.36 & 2.25 & 0.10 \\
\hline 20 & 2.35 & 2.02 & 0.33 \\
\hline 21 & 2.41 & 2.22 & 0.18 \\
\hline 22 & 2.33 & 2.27 & 0.05 \\
\hline 23 & 2.36 & 2.21 & 0.14 \\
\hline 24 & 1.56 & 1.77 & -0.21 \\
\hline 25 & 1.23 & 1.18 & 0.04 \\
\hline 26 & 2.02 & 1.72 & 0.30 \\
\hline 27 & 1.87 & 1.69 & 0.17 \\
\hline 28 & 1.11 & 1.19 & -0.08 \\
\hline 29 & 1.69 & 1.67 & 0.01 \\
\hline 30 & 1.60 & 1.64 & -0.03 \\
\hline 31 & 1.44 & 1.65 & -0.20 \\
\hline 32 & 0.95 & 1.51 & -0.56 \\
\hline 33 & 1.87 & 1.33 & 0.53 \\
\hline 34 & 2.47 & 2.24 & 0.23 \\
\hline 35 & 2.50 & 2.28 & 0.22 \\
\hline 36 & 2.23 & 2.28 & -0.05 \\
\hline 37 & 2.20 & 2.35 & -0.15 \\
\hline 38 & 1.77 & 2.02 & -0.25 \\
\hline 39 & 2.04 & 1.91 & 0.12 \\
\hline 40 & 1.60 & 1.70 & -0.10 \\
\hline 41 & 1.84 & 1.64 & 0.19 \\
\hline 42 & 1.44 & 1.42 & 0.01 \\
\hline 43 & 1.87 & 1.61 & 0.25 \\
\hline 44 & 2.09 & 2.28 & -0.18 \\
\hline 45 & 2.04 & 2.34 & -0.30 \\
\hline
\end{tabular}

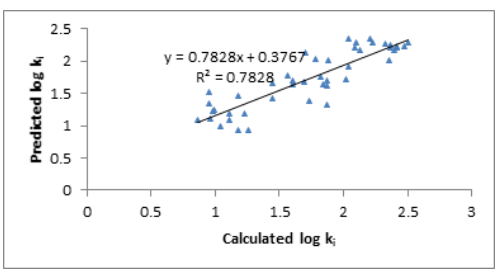

Fig 1: Graph plotted between predicted $\log \mathrm{KI}$ and calculated $\log$ Ki

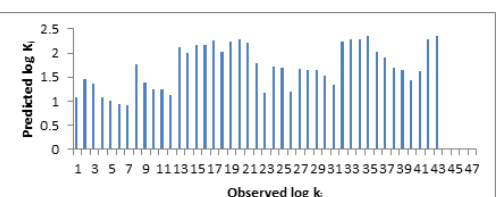

Fig 2: Graph plotted between predicted Residual and obrseved log KI

\section{REFERENCES}

1. Hassan MU, Chohan ZH, Scozzafava A, Supuran CT, J. Enz. Inh. Med. Chem. 19 (2004)

2. Rusconi S, Innocenti A, Vullo D, Mastrolorenzo A, Scozzafava A, Supuran C T, Bioorg. Med. Chem. Lett., 14 (2004) 5763 .

3. Scozzafava A, Menabuoni L, Mincione F, Briganti F, Mincione G, Supuran C T, J. Med. Chem. 2000, 43 (2004) 4542

4. Scozzafava A, Menabuoni L, Mincione F, Supuran CT, J. Med. Chem. 45 (2002) 1466. Debnath AK, In Combinatorial Library Design and Evaluation, Principles Software Tools and Applications in Drug Discovery, Ghose AK, Viswanadhan VN, Eds.; Marcel Dekker New York (2001)pp 73-129.

6. Melagraki G, Afantitis A, Bioorganic \& Medicinal Chemistry 14 (2006) 1108-1114.

7. Hansch C, Sammes PG, Taylor JB, Comprehensive Medicinal Chemistry, Vol. 2, Pergamon Press Oxford, (1990) chap. 7.1.

8. Kanda Y, Kawanishi Y, Oda K, Sakata, T, Mihara S, Asakura, K, Kanemasa T, Ninomiya M, Fujimoto M, Kanoike T, Bioorg. Med. Chem., 9 (2001) 897-907.

9. Chibale K, Haupt H, Kendrick H, Yardley V, Saravanamuthu A, Fairlamb AH, Croft SL, Bioorg. Med. Chem. Lett., 11 (2001) 2655-2657.

10. Rahavi Ezabadi I, Camoutsis C, Zoumpoulakis P, Geronikaki A, Sokovic M, Glamocilija J, Ciric A, Bioorg. Med. Chem. (2008), 16 (2001) 1150-1161.

11. Kennedy JF, Thorley M, Pharmaceutical Substances, 3rd ed., Kleeman A, Engel J, Kutscher B, ReichertD,(Eds.), Thieme, Stuttgart, (1999).

12. Serradeil Le Gal C, Cardiovascular Drug Rev. 19 (2001) 201-214

13. Natarajan A, Guo Y, Harbinski F, Fan YH, Chen H, Luus L, Diercks J, Aktas H, Chorev M, Halperin JA J. Med. Chem., 47 (2004) 4979-4982

14. Vullo D, De Luca V, Scozzafava A, Carginale V, Rossi M, Supuran CT, Capasso C, Bioorg. Med. Chem., 21 (2013) 4521-4525.

15. Wilson CO, Gisvold O, Block JH, Wilson and Gisvold's Textbook of Organic Medicinal and Pharmaceutical Chemistry, 11 th ed., Block J, Beale JM, (Eds.), Lippincott Williams and Wilkins, Philadelphia (2004).

16. Levin JI, Chen JM, Du MT, Nelson FC, Killar LM, Skala S, Sung A, Jin G, Cowling R, Barone D, March CJ, Mohler KM, Black, RA, Skotnicki JS Bioorg. Med. Chem. Lett., 